\title{
COMPOSIÇÃO, ESPECTRO BIOLÓGICO E SÍNDROMES DE DISPERSÃO DA VEGETAÇão de um INSELbERGUe No domínio da CAATINGa, CEARÁ
}

\author{
Francisca Soares Araújo ${ }^{1,2}$, Rosilane Ferreira Oliveira1 \& Luiz Wilson Lima-Verde ${ }^{1}$
}

\begin{abstract}
Resumo
(Composição, espectro biológico e síndromes de dispersão da vegetação de um inselbergue no domínio da caatinga, Ceará) Comunidades de plantas sobre afloramentos rochosos no semi-árido brasileiro ainda são pouco conhecidas para a ciência. O município de Quixadá, no semi-árido cearense, destaca-se pela grande concentração de elevações de ilhas rochosas. Este estudo tem por objetivos levantar e analisar a composição, o espectro biológico e as síndromes de dispersão das espécies que ocorrem em um inselbergue no município de Quixadá, Ceará, e a similaridade florística deste com as espécies da vegetação circundante. Em 2000, foram realizadas coletas mensais de espécies e classificadas quanto às formas de vida e síndromes de dispersão em uma área situada a $4^{\circ} 57^{\prime} \mathrm{S}$ e $39^{\circ} 01^{\prime} \mathrm{W}$ e $270 \mathrm{~m}$ de altitude. A similaridade da flora com o entorno foi analisada através do índice de Jaccard. Foram inventariadas 77 espécies, 66 gêneros e 36 famílias. As porcentagens do espectro biológico foram: terófitos $(44,2)$, fanerófitos $(24,7)$, caméfitos $(14,6)$, hemicriptófitos $(13,4)$, geófitos $(2,6)$ e aerófitos $(1,2)$ e as do espectro de dispersão: anemocoria (49), autocoria (35) e zoocoria (16). A similaridade com flora do entorno foi de $13 \%$ (21 espécies), demonstrando que a caatinga local é a potencial fonte de propágulos. Os terófitos são as formas de vida dominante nos inselbergues de regiões áridas e semiáridas, cuja flora também é predominantemente dispersa por fatores abióticos, principalmente o vento. Assim, este estudo confirma o padrão esperado para os atributos síndromes de dispersão e espectro biológico da vegetação sob climas áridos e semi-áridos.
\end{abstract}

Palavras-chave: afloramentos rochosos, semi-árido, flora, forma de vida, caatinga.

\section{Abstract}

(Composition, biological spectrum and dispersal syndromes of the vegetation of an Inselberg in the domain of caatinga, Ceará) Plant communities on rock outcrops in brazilian semiarid are still largely unknown to science. The country Quixadá, in the semiarid of the state of Ceará, is distinguished by the great concentration of rock outcrops. This study aims to know the composition, the life-form and the strategies of dispersal of the species in an inselberg the country of Quixadá and the similarity with surrounding caatinga. In the 2000 year, monthly collects of the flora were carried out and the life-forms and dispersal syndromes were classified in an area situated on $4^{\circ} 57^{\prime} \mathrm{S}$ and $39^{\circ} 01^{\prime} \mathrm{W}, 270 \mathrm{~m}$. a.s.l. The similarity of the flora of the surrounding was analyzed through the Jaccard index. Seventy-seven species, 66 genera and 36 families had been inventoried. The percentages of the life-form spectrum and the dispersal spectrum represented were: therophytes $(44,2)$, phanerophytes $(24,7)$, chamaephytes $(14,6)$, hemicryptophytes $(13,4)$, geophytes $(2,6)$, aerophytes $(1,2)$ and anemocoric (49), autocoric (35) and zoocoric (16). The similarity with the flora of the caatinga surrounding was 13\% (21 species), what it demonstrated that the vegetation surrounding is the main source of propagules. The therophytes are the dominant life-form in inselbergs of semi-arid regions, whose flora also is predominantly dispersed for abiotic factors, mainly the wind. This study confirms the expected patterns about dispersal and life-form spectrum of the arid and semiarid vegetation.

Key words: rock outcrops, semiarid, flora, life form, caatinga.

\section{INTRODUÇÃO}

Comunidades de plantas sobre afloramentos rochosos são comumente encontradas no semiárido brasileiro. Borhardt usou o termo inselbergue para denominar as elevações de ilhas rochosas que ocorrem em regiões de clima árido (Guerra
1978). Entretanto, Barthlott et al. (1993) e Porembski et al. (1997) referem-se ao termo inselbergue indistintamente para denominar rochas solitárias, monolíticas, ou grupos de montanhas rochosas de granito ou gnaisse, que se elevam abruptamente na paisagem plana, em regiões de

Artigo recebido em 09/2007. Aceito para publicação em 07/2008.

${ }^{1}$ Departamento de Biologia, Bloco 906, Centro de Ciências, Universidade Federal do Ceará, Campus do Pici, C.P. 6021, 60455-970, Fortaleza, CE, Brasil.

${ }^{2}$ Autor para corresppondência: tchesca@ufc.br 
climas semi-áridos e úmidos. Essas ilhas rochosas concentram-se nos antigos escudos cristalinos da América do Sul (e. q., a Guiana e o Brasil) e da África (Barthlott et al. 1993). Apresentam condições pedológicas e microclimáticas (estresse hídrico, insolação e temperaturas elevadas) bem diferenciadas das do seu entorno (Barthlott et al. 1993; Ibisch et al. 1995). Assim, segundo Porembski et al. (1994, 1998), as plantas que colonizam esses habitats rochosos apresentam adaptações à falta de água e ao substrato pouco desenvolvido, mesmo naqueles situados no domínio de climas úmidos.

A vegetação que ocorre nos inselbergues azonais, segundo Porembski et al. (1997), apresenta composição florística e distribuição espacial de espécies bastante heterogêneas devido aos vários tipos de habitats que podem ocorrer, a saber: a) depressões rasas cobertas por liquens; b) tanques de rocha rasos, com água sazonal, onde predominam ervas terofíticas (Poaceae, Cyperaceae e Eriocaulaceae); c) tanques de rochas profundos, com substrato pouco desenvolvido, onde predominam monocotiledôneas hemicriptofíticas/camefíticas (Bromeliaceae, Cyperaceae e Velloziaceae), além de Cactaceae camefíticas; e) fissuras na rocha, onde ocorrem os fanerófitos (arbustos e árvores de pequeno porte). Bromeliaceae, Cactaceae, Cyperaceae, Orchidaceae, Poaceae e Velloziaceae estão entre as famílias que mais se destacam nas ilhas de vegetação sobre afloramentos rochosos neotropicais (Ibisch et al. 1995).

As síndromes abióticas, principalmente a anemocoria, representam mais de $80 \%$ da flora dos inselbergues (Porembski et al. 1998; Burke et al. 1998), inclusive naqueles situados sob climas úmidos há predomínio de síndromes abióticas (Porembski et al. 1998). Isso reflete a aridez microclimática dessas ilhas rochosas em relação ao entorno (Barthlott et al. 1993; Ibisch et al. 1995), pois representam habitats sob maior exposição aos ventos e à insolação (Barthlott et al. 1993; Ibisch et al. 1995; Porembski et al. 1998) e, assim, favorecem a entrada e a colonização de espécies de síndromes abióticas, cuja fonte principal de propágulos, como ressaltado por Scarano (2007), é a vegetação circundante.
No semi-árido brasileiro, em estudos realizados na caatinga sentido restrito, na savana decíduo espinhosa (Araújo et al. 2005a), predominam espécies de dispersão abiótica (Griz \& Machado 2001; Barbosa et al. 2002) e terófito é a forma de vida que apresenta maior riqueza de espécies (Araújo et al. 2005b; Costa et al. 2007; Mamede \& Araújo 2008; Rodal et al. 2005). Terófito é a forma de vida dominante entre as espécies de vegetação sob climas áridos e semiáridos (Kovács-Lang et al. 2000; Raunkiaer 1934; van Rooyen et al. 1990). Em consequiência, terófitos de dispersão abióticas predominam entre as espécies que colonizam habitats sobre afloramentos rochosos em climas áridos e semiáridos (Krieger et al. 2000; Porembski et al. 1995, 1996, 1998; Sarthou \& Villiers 1998).

Entretanto, em dois estudos de ilhas de vegetação sobre afloramentos no semi-árido da Bahia (França et al. 2005, 2006), fanerófito foi a forma de vida mais representativa em número de espécies.

Porém, comunidades de plantas sobre afloramentos rochosos no semi-árido brasileiro ainda são pouco conhecidas para a ciência. E, embora o clima dominante seja semi-árido, em uma das faces de cada área estudada, como descrito por França et al. (1997), ocorre floresta semi-decídua, o que caracteriza a ocorrência local de ambiente mais úmido e, assim, justifica o domínio de fanerófitos.

O inselbergue aqui estudado não apresenta condições climáticas locais diferenciadas das do clima dominante regionalmente. Neste caso, infere-se que a composição de espécies é dominada por aquelas com dispersão abiótica e forma de vida terofítica, padrão para climas áridos e semi-áridos, já registrado inclusive na vegetação de caatinga. Devido à distância geográfica e diferenças locais de umidade, a composição de espécies do Inselbergue de Quixadá deve ser mais similar à da vegetação circundante do que à dos afloramentos estudados na Bahia.

Assim, este trabalho tem por objetivos conhecer e analisar a composição, o espectro biológico e as síndromes de dispersão das espécies que ocorrem em um monólito no município de Quixadá, Ceará, e a similaridade florística deste 
com as espécies da vegetação circundante. Além disso, será verificado se os espectros de dispersão e de forma de vida encontrados em Quixadá confirmam os padrões encontrados em inselbergues de outras regiões climáticas áridas e semi-áridas.

\section{Material e Métodos}

\section{Localização e caracterização da área de estudo}

O município de Quixadá, localizado no sertão central do estado do Ceará e situado a cerca de $200 \mathrm{~km}$ ao sul de Fortaleza, destacase pela grande concentração de elevações de ilhas rochosas monolíticas (Fig. 1) (Souza 1988). O monólito estudado está localizado no sítio Santa Luzia, arredores da cidade de Quixadá (457’20,6" S e 3901'28' W, 270 m de altitude) e apresenta cerca de $70 \mathrm{~m}$ de altura entre a base e o topo.

A precipitação média anual na sede municipal de Quixadá é de 732,8 mm, concentrada nos meses de fevereiro a maio $(79,6 \%$ ), atinge o máximo de $178,3 \mathrm{~mm}$ no mês de março. O período mais seco estende-se de junho a dezembro. A temperatura média anual estimada por regressão é de $26,6^{\circ} \mathrm{C}$, variando de $25,2^{\circ} \mathrm{C}$, no período mais frio, nos meses de junho e julho, a $27,4^{\circ} \mathrm{C}$, em novembro (SUDENE 1982). Os dados pluviométricos encontram-se disponíveis no sítio da Fundação Cearense de Meteorologia (FUNCEME 2007).

\section{Levantamento florístico}

Durante todo o ano de 2000 foram realizadas coletas mensais de plantas vasculares: plantas inteiras herbáceas e ramos reprodutivos das lenhosas (botões florais, flores e/ou frutos). As coletas foram realizadas em toda a área do monólito, exceto na base por não mais haver vegetação (Fig. 1), nos habitats depressões, fissuras e rocha exposta, independente da organização espacial da vegetação, se em ilhas ou não. Para cada tipo de habitat (depressão, fissuras e rocha exposta) sobre o inselbergue foram caracterizadas visualmente as espécies dominantes.
No momento da coleta foram determinadas a forma de vida das espécies coletadas (Raunkiaer 1934) e a síndrome de dispersão dos espécimes com fruto (van der Pijl 1982). Quando não foi possível coletar a planta com fruto, a síndrome foi definida a partir da análise de material de herbário ou de informações na literatura.

Foram consideradas espécies terofíticas as que não apresentam sistema de brotamento no corpo da planta, sistema subterrâneo fasciculado ou ramificado, sem qualquer tipo de estrutura perene, como rizoma, tubérculo ou bulbo. Terófitos são plantas anuais, dependem de uma estação favorável para o desenvolvimento e, assim, completam o ciclo vital, isto é, após a produção de sementes, morrem durante a estação desfavorável (Raunkiaer 1934). Além das formas de vida de Raunkiaer (1934), foi acrescentada a forma de vida aerófito. Segundo Galán de Mera et al. (1999), aerófitos são espécies herbáceas perenes com sistema radicular atrofiado, apresentam gemas de crescimento acima da superfície do solo, em geral, sobre a rocha exposta.

Todo o material botânico foi herborizado segundo as normas de Mori et al. (1989) e incorporado ao acervo herbário EAC. O material coletado foi identificado até o nível de espécie, quando possível, com auxílio de literatura específica e comparação com o material determinado por especialistas, depositado no herbário EAC. Quando necessário, o material foi enviado a especialistas para identificação e/ou confirmação. Todas as espécies coletadas foram listadas por ordem alfabética de família, segundo o sistema de Cronquist (1981), exceto para Leguminosae (Lewis et al. 2005). A abreviação dos autores das espécies foi verificada no Brummitt \& Powell (1992).

\section{Análise do espectro biológico, síndromes de dispersão e similaridade florística}

Foram calculadas as porcentagens de espécies por formas de vidas e por síndromes de dispersão. Ambos, espectro de dispersão e de formas de vida, foram comparados com os de trabalhos realizados em elevações de ilhas rochosas de outras regiões. 

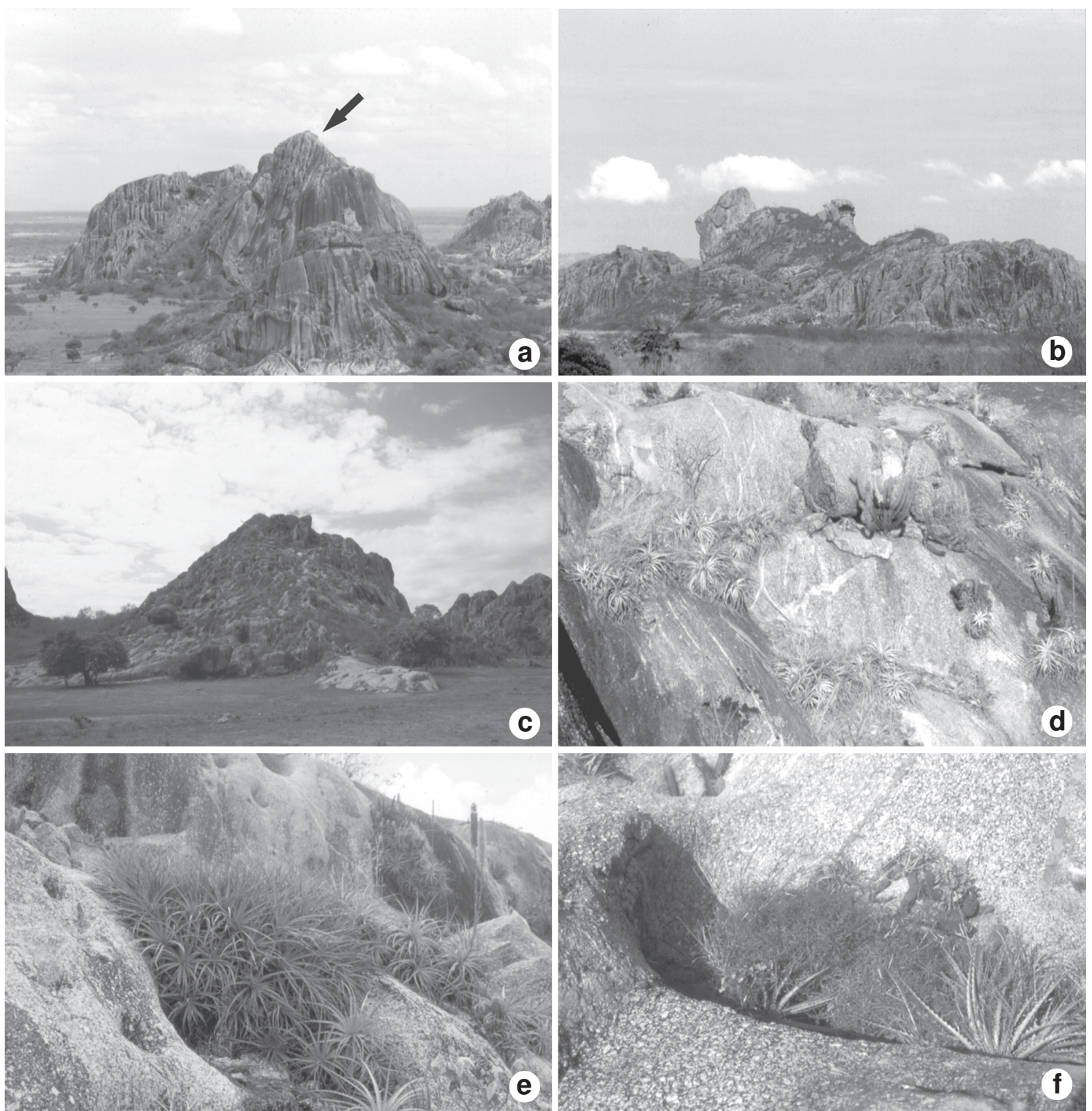

Figura 1 - a-c. Aspecto geral de alguns inselbergues em Quixadá, Ceará. A seta na foto a indica o monólito estudado; d. plantas sobre o hábitat rocha exposta; e-f. plantas sobre o hábitat depressão.

A área circundante do referido inselbergue é ocupada por atividades agropastoris e não há, nas proximidades imediatas, remanescente de vegetação para levantamento da flora do entorno e, assim, compará-las entre si. Para comparação com a flora do entorno, foi utilizado o estudo realizado por Costa et al. (2007) em uma área de caatinga arbustiva-arbórea também no município de Quixadá, porém distante cerca de $22 \mathrm{~km}$. Aqueles autores coletaram todas as espécies presentes em uma área de cerca de dois hectares e calcularam o espectro biológico.
O índice de similaridade de Jaccard (Krebs 1989) foi calculado para comparar a composição de espécies do monólito estudado com a da caatinga circundante estudada por Costa et al. (2007). Também foi verificada a ocorrência das espécies da área estudada em Quixadá em listas florísticas de ilhas de vegetação sobre afloramentos rochosos do semi-árido baiano (França et al. 2005, 2006). Não foi analisado o trabalho de França et al. (1997), pois trata-se da lista florística dos mesmos locais de França et al. $(2005,2006)$ e inclui também a flora da base circundante daqueles inselbergues. 


\section{Resultados e Discussão}

Foram encontradas 77 espécies pertencentes a 36 famílias (Tab. 1). As cinco famílias que apresentaram maior riqueza de espécies somaram, em conjunto, $44 \%$ das espécies inventariadas: Leguminosae (11 spp.), Poaceae (10 spp.), Euphorbiaceae (cinco spp.), Asteraceae e Convolvulaceae (quatro spp.). Os gêneros com maior número de espécies foram Portulaca, com três espécies, Campomanesia, Croton, Cyperus, Digitaria, Evolvulus, Mimosa, Paspalum e Pilosocereus com duas espécies cada (Tab. 1).

Poaceae está entre as famílias mais ricas de inselbergues, principalmente naqueles situados em climas mais secos (Tab. 2). A alta riqueza de Poaceae no monólito de Quixadá corrobora as afirmações de Ibisch et al. (1995) sobre ser Poaceae uma das famílias de maior diversidade nos inselbergues tropicais. Porém, a alta diversidade de Poaceae possivelmente está associada a afloramentos rochosos sob climas mais secos, pois em climas mais úmidos, Bromeliaceae e Orchidaceae estão entre as famílias mais diversificadas (Tab. 2). Em Quixadá, Bromeliaceae e Orchidaceae foram representadas por apenas duas e uma espécie, respectivamente. $\mathrm{O}$ fato da alta riqueza de Orchidaceae e baixa porcentagem de terófitos (França et al. 2005) não estar no padrão já registrado para climas áridos e semi-áridos, reflete condições locais de maior umidade como a ocorrência de floresta semi-decídua em uma das faces daquela área de estudo.

$\mathrm{Na}$ análise comparativa entre os estudos já realizados em afloramentos rochosos sob diversos tipos climáticos, embora haja grandes diferenças de riqueza e de condições ambientais (Tab. 2), observa-se que a vegetação sobre afloramentos rochosos do semi-árido brasileiro apresenta alta riqueza de espécies. Como ressaltado por Scarano (2007), isso demonstra a importância de estudos fitogeográficos e da biologia e ecologia dessas espécies e de ações para a conservação dessas áreas.

A vegetação do inselbergue de Quixadá ocorre geralmente em agrupamentos ilhas de tamanhos variados, nem sempre isolados (Fig. 1d-f), sobre três tipos de habitats: 1) depressões rasas e profundas distribuídas em todo o inselbergue, onde nas mais rasas, dominam populações de Mandevilla tenuifolia, Cyperus uncinulatus, Pennisetum pedicellatum e Aristida setifolia e nas mais profundas, populações de bromélias e cactos, respectivamente, Encholirium spectabile, Pilosocereus gounellei e $P$. cf. piauhiensis; 2) fissuras na rocha de comprimentos e profundidades variadas, localizadas predominantemente na porção mediana do monólito, em que se encontram arbustos e pequenas árvores, tais como: Combretum leprosum, Cochlospermum vitifolium, Commiphora leptophloeos, Cordia insignis, Croton lundianus, $C$. moritibensis e Lantana camara e 3) rocha exposta com agregados pouco freqüentes de populações de Tillansia sp.

As famílias Cactaceae e Bromeliaceae, além de numerosas em espécie na caatinga como um todo, são estruturalmente dominantes, em abundância e freqüência, nos inselbergues de Quixadá. A família Bromeliaceae também tem grande importância estrutural em outros afloramentos rochosos da América (Tab. 2).

A similaridade de espécies com a flora do entorno foi de $13 \%$, o que corresponde à ocorrência em comum de 21 espécies, principalmente de terófitos. Em relação aos outros inselbergues do semi-árido brasileiro, das 77 espécies registradas em Quixadá, apenas quatro ocorreram no estudo de França et al. (2005) e duas no de França et al. (2006), na Bahia (Tab. 1). Embora sejam ambientes rochosos sob o mesmo domínio climático regional, a baixa ocorrência de espécies em comum entre este estudo e os da Bahia, reflete as distâncias geográficas e as características da vegetação circundante, que nos da Bahia é uma floresta semi-decídua (França et al. 1997) e, indica, como ressaltado por Scarano (2007), que a vegetação circundante é a principal fonte de propágulos desses afloramentos rochosos.

O espectro de dispersão (Tab. 3) foi representado por 38 espécies anemocóricas (49\%), 27 autocóricas (35\%) e 12 zoocóricas (16\%). A maioria das espécies autocóricas foi representada 
Tabela 1 - Famílias com as respectivas espécies em ordem alfabética; número do coletor principal Oliveira, R. F. (NC); habitats de ocorrência: fissura (F), depressão (D) e rocha exposta (R); formas de vida (FV): fanerófito (fn), caméfito (ch), hemicriptófito (hm), criptófito geófito (cg), terófito (th), aerófito (ar) e síndromes de dispersão (SD): anemocórica (an), zoocórica (zo) e autocórica (au), encontradas no monólito do município de Quixadá, Ceará, e sua ocorrência na caatinga do entorno (1- Costa et al. 2007) e em inselbergues da caatinga baiana (2- França et al. 2005 e 3- França et al. 2006).

\begin{tabular}{|c|c|c|c|c|c|c|c|c|c|c|}
\hline \multirow[b]{2}{*}{ Famílias } & \multirow[b]{2}{*}{ Espécies } & \multicolumn{4}{|c|}{ Hábitat } & \multirow[t]{2}{*}{ FV } & \multirow[t]{2}{*}{ SD } & \multicolumn{3}{|c|}{$\begin{array}{l}\text { Locais de } \\
\text { ocorrência }\end{array}$} \\
\hline & & $\mathrm{NC}$ & $\mathbf{F}$ & $\mathbf{D}$ & $\mathbf{R}$ & & & 1 & 2 & 3 \\
\hline Amaranthaceae & Froelichia lanata Moench & 32 & & $\mathrm{x}$ & & $\mathrm{hm}$ & an & & & \\
\hline Amaryllidaceae & Zephyranthes cf. cearensis A.DC. & 25 & & $\mathrm{x}$ & & $\mathrm{cg}$ & an & & & \\
\hline \multirow[t]{2}{*}{ Apocynaceae } & Allamanda blanchetii Müll. Arg. & 7 & $\mathrm{x}$ & $\mathrm{x}$ & & $\mathrm{hm}$ & an & $\mathrm{x}$ & & \\
\hline & $\begin{array}{l}\text { Mandevilla tenuifolia (J.C. Mikan) } \\
\text { Woodson }\end{array}$ & 10 & & $\mathrm{x}$ & & $\mathrm{cg}$ & an & & & \\
\hline Araceae & Philodendron acutatum Schott & 17 & & $\mathrm{x}$ & & $\mathrm{hm}$ & zo & & & \\
\hline Asclepiadaceae & $\begin{array}{l}\text { Marsdenia megalantha Goyder } \\
\text { \& Morillo }\end{array}$ & 57 & & $\mathrm{x}$ & & fh & an & & & \\
\hline \multirow[t]{4}{*}{ Asteraceae } & Delilia biflora (L.) Kuntze & 1229 & & $\mathrm{x}$ & & th & an & & & \\
\hline & $\begin{array}{l}\text { Pithecoseris pacourinoides } \\
\text { Mart. ex DC. }\end{array}$ & 126 & & $\mathrm{x}$ & & th & an & & & \\
\hline & Vernonia grisea Baker & 121 & & $\mathrm{x}$ & & th & an & & & \\
\hline & Tridax procumbens $\mathrm{L}$. & 86 & & $\mathrm{x}$ & & th & an & & & \\
\hline Bignoniaceae & $\begin{array}{l}\text { Pithecoctenium crucigerum (L.) } \\
\text { A.H. Gentry }\end{array}$ & 5 & $\mathrm{x}$ & & & fh & an & & & \\
\hline \multirow[t]{2}{*}{ Boraginaceae } & Cordia oncocalyx Allemão & 26 & $\mathrm{x}$ & & & fh & an & $\mathrm{x}$ & & \\
\hline & Cordia insignis Cham. & 90 & $\mathrm{x}$ & & & fh & an & & & \\
\hline \multirow[t]{2}{*}{ Bromeliaceae } & $\begin{array}{l}\text { Encholirium spectabile Mart. } \\
\text { ex Schult. \& Schult.f. }\end{array}$ & 1 & & $\mathrm{x}$ & & $\mathrm{hm}$ & an & & $\mathrm{x}$ & $\mathrm{x}$ \\
\hline & Tillandsia $\mathrm{sp}$ & 135 & & & $\mathrm{x}$ & ar & an & & & \\
\hline Burseraceae & $\begin{array}{l}\text { Commiphora leptopholoeos } \\
\text { (Mart.) Gillett }\end{array}$ & 41 & $\mathrm{x}$ & & & fh & zo & $\mathrm{x}$ & & \\
\hline \multirow{3}{*}{ Cactaceae } & Opuntia inamoema K. Schum. & 22 & & $\mathrm{x}$ & & $\mathrm{ch}$ & zo & & & \\
\hline & $\begin{array}{l}\text { Pilosocereus gounellei (F.A.C. } \\
\text { Weber) Byles \& G.D. Rowley }\end{array}$ & 23 & & $\mathrm{x}$ & & fh & zo & & & $\mathrm{x}$ \\
\hline & $\begin{array}{l}\text { Pilosocereus cf. piauhyensis (Gürk) } \\
\text { Byles \& G.D. Rowley }\end{array}$ & 24 & $\mathrm{x}$ & $\mathrm{x}$ & & fh & zo & & & \\
\hline Cochlospermaceae & $\begin{array}{l}\text { Cochlospermum vitifolium (Willd.) } \\
\text { Spreng. }\end{array}$ & 118 & $\mathrm{x}$ & & & fh & $\mathrm{au}$ & $\mathrm{x}$ & & \\
\hline Combretaceae & Combretum leprosum Mart. & 137 & $\mathrm{x}$ & & & fh & an & $\mathrm{x}$ & & \\
\hline \multirow[t]{2}{*}{ Commelinaceae } & Commelina sp. & 84 & & $\mathrm{x}$ & & th & $\mathrm{au}$ & & & \\
\hline & $\begin{array}{l}\text { Callisia cf. filiformis (M. Martens } \\
\text { \& Galeotti) D. R. Hunt. }\end{array}$ & 82 & & $\mathrm{x}$ & & th & $\mathrm{au}$ & $\mathrm{x}$ & & \\
\hline \multirow[t]{4}{*}{ Convolvulaceae } & Evolvulus filipes Mart. & 97 & & $\mathrm{x}$ & & th & an & & & \\
\hline & Evolvulus sp. & 109 & & $\mathrm{x}$ & & th & an & & & \\
\hline & Ipomea rosea Choisy & 91 & $\mathrm{x}$ & $\mathrm{x}$ & & $\mathrm{ch}$ & an & $\mathrm{x}$ & & \\
\hline & Jacquemontia sp. & 106 & $\mathrm{x}$ & $\mathrm{x}$ & & $\mathrm{hm}$ & an & & & \\
\hline \multirow[t]{2}{*}{ Cyperaceae } & $\begin{array}{l}\text { Bulbostylis cf. hirtella (Schrad. } \\
\text { ex Schult.) Nees ex Urb. }\end{array}$ & 61 & & $\mathrm{x}$ & & th & an & & & \\
\hline & Cyperus cf. schomburgkianus Ness. & 63 & & $\mathrm{x}$ & & th & an & & & \\
\hline
\end{tabular}




\begin{tabular}{|c|c|c|c|c|c|c|c|c|c|c|}
\hline \multirow[b]{2}{*}{ Famílias } & \multirow[b]{2}{*}{ Espécies } & \multirow[b]{2}{*}{$\mathrm{NC}$} & \multicolumn{3}{|c|}{ Hábitat } & \multirow[t]{2}{*}{ FV } & \multirow[t]{2}{*}{ SD } & \multicolumn{3}{|c|}{$\begin{array}{l}\text { Locais de } \\
\text { ocorrência }\end{array}$} \\
\hline & & & $\mathbf{F}$ & $\mathbf{D}$ & $\mathbf{R}$ & & & 1 & 2 & 3 \\
\hline & Cyperus uncinulatus Shard ex Ness & 89 & & $\mathrm{x}$ & & th & an & $\mathrm{x}$ & & \\
\hline Curcubitaceae & Cayaponia racemosa (Mill.) Cogn. & 100 & $\mathrm{x}$ & $\mathrm{x}$ & & $\mathrm{ch}$ & zo & $\mathrm{x}$ & & \\
\hline Eriocaulaceae & Paepalanthus sp. & 4 & & $\mathrm{x}$ & & th & an & & & \\
\hline \multirow[t]{5}{*}{ Euphorbiaceae } & Cnidosculus urens (L.) Arthur & 14 & $\mathrm{x}$ & $\mathrm{x}$ & & fh & $\mathrm{au}$ & & $\mathrm{x}$ & \\
\hline & Croton moritibensis Baill. & 50 & $\mathrm{x}$ & $\mathrm{x}$ & & fh & $\mathrm{au}$ & $\mathrm{x}$ & & \\
\hline & Croton lundianus (Didr.) Müll. Arg. & 28 & $\mathrm{x}$ & $\mathrm{x}$ & & fh & $\mathrm{au}$ & & & \\
\hline & Dalechampia pernambucensis Baill. & 9 & $\mathrm{x}$ & $\mathrm{x}$ & & th & $\mathrm{au}$ & $\mathrm{x}$ & & \\
\hline & Jatropha pholiana Müll. Arg. & 18 & $\mathrm{x}$ & $\mathrm{x}$ & & fh & $\mathrm{au}$ & & & \\
\hline Gentianaceae & $\begin{array}{l}\text { Schultessia guianensis (Aubl.) } \\
\text { Malme }\end{array}$ & 102 & & $\mathrm{x}$ & & th & $\mathrm{au}$ & & & \\
\hline Gesneriaceae & $\begin{array}{l}\text { Sinnigia incarnata (Aubl.) } \\
\text { D.L. Denham }\end{array}$ & 31 & & $\mathrm{x}$ & & $\mathrm{hm}$ & $\mathrm{au}$ & & & \\
\hline Lamiaceae & $\begin{array}{l}\text { Marsypianthes chamaedrys } \\
\text { (Vehl) Kuntze }\end{array}$ & 96 & & $\mathrm{x}$ & & th & $\mathrm{au}$ & $\mathrm{x}$ & & \\
\hline Leg. Caesalpinioideae & $\begin{array}{l}\text { Bauhinia pentandra (Bong.) } \\
\text { Vogel ex Steud. }\end{array}$ & 65 & $\mathrm{x}$ & & & fh & $\mathrm{au}$ & & & \\
\hline \multirow[t]{2}{*}{ Leg. Mimosoideae } & Mimosa camporum Benth. & 68 & $\mathrm{x}$ & $\mathrm{x}$ & & th & $\mathrm{au}$ & & & \\
\hline & Mimosa invisa Mart. ex Colla & 134 & $\mathrm{x}$ & $\mathrm{x}$ & & fh & zo & & & \\
\hline \multirow[t]{8}{*}{ Leg. Papilionioideae } & Aeschynomene scabra G. Don & 104 & & $\mathrm{x}$ & & th & $\mathrm{au}$ & & & \\
\hline & Centrosema venosum Mart. ex Benth. & 92 & $\mathrm{x}$ & $\mathrm{x}$ & & $\mathrm{ch}$ & $\mathrm{au}$ & & & \\
\hline & $\begin{array}{l}\text { Crotalaria } \text { cf. holosericea Nees } \\
\text { \& C. Mart. }\end{array}$ & 80 & $\mathrm{x}$ & $\mathrm{x}$ & & fh & $\mathrm{au}$ & $\mathrm{x}$ & $\mathrm{x}$ & \\
\hline & Desmodium glabrum (Mill.) DC. & 114 & $\mathrm{x}$ & $\mathrm{x}$ & & $\mathrm{hm}$ & $\mathrm{au}$ & & & \\
\hline & Indigofera suffruticosa Mill. & 53 & $\mathrm{x}$ & $\mathrm{x}$ & & $\mathrm{ch}$ & $\mathrm{au}$ & & & \\
\hline & $\begin{array}{l}\text { Macroptilium martii (Benth.) } \\
\text { Maréchal \& Baudet }\end{array}$ & 148 & $\mathrm{x}$ & $\mathrm{x}$ & & th & $\mathrm{au}$ & $\mathrm{x}$ & & \\
\hline & Stylosanthes humilis Kunth & 98 & & $\mathrm{x}$ & & th & $\mathrm{au}$ & $\mathrm{x}$ & & \\
\hline & Zornia aff. sericea Moric. & 64 & & $\mathrm{x}$ & & th & $\mathrm{au}$ & & & \\
\hline \multirow{2}{*}{ Myrtaceae } & Campomanesia sp.1 & 150 & $\mathrm{x}$ & & & fh & zo & & & \\
\hline & Campomanesia sp.2 & 151 & $\mathrm{x}$ & & & fh & zo & & & \\
\hline Onagraceae & Ludwigia sp. & 81 & $\mathrm{x}$ & $\mathrm{x}$ & & $\mathrm{ch}$ & $\mathrm{au}$ & & & \\
\hline Orchidaceae & Cyrtopodium sp. & 48 & & $\mathrm{x}$ & & $\mathrm{ch}$ & an & & & \\
\hline Passifloraceae & Passiflora foetida $\mathrm{L}$. & 51 & & $\mathrm{x}$ & & $\mathrm{ch}$ & zo & $\mathrm{x}$ & & \\
\hline \multirow[t]{10}{*}{ Poaceae } & Andropogon sp. & 40 & & $\mathrm{x}$ & & th & an & & & \\
\hline & Aristida setifolia Kunth & 99 & & $\mathrm{x}$ & & th & an & & & \\
\hline & Brachiaria fasciculata (Sw.) Parodi & 48 & & $\mathrm{x}$ & & th & an & & & \\
\hline & Digitaria ciliaris (Retz.) Koeler & 140 & & $\mathrm{x}$ & & th & an & & & \\
\hline & Digitaria sp. & 74 & & $\mathrm{x}$ & & th & an & & & \\
\hline & Melinis repens (Willd.) Zizka & 35 & & $\mathrm{x}$ & & th & an & & $\mathrm{x}$ & \\
\hline & Panicum trichoides Sw. & 131 & & $\mathrm{x}$ & & th & an & $\mathrm{x}$ & & \\
\hline & Paspalum sp.1 & 71 & & $\mathrm{x}$ & & th & an & & & \\
\hline & Paspalum sp. 2 & 112 & & $\mathrm{x}$ & & th & an & & & \\
\hline & Pennisetum pedicellatum Trin. & 85 & & $\mathrm{x}$ & & th & an & & & \\
\hline \multirow[t]{3}{*}{ Portulacaceae } & Portulaca cf. halimoides L. & 45 & & $\mathrm{x}$ & & $\mathrm{hm}$ & an & & & \\
\hline & Portulaca sp.1 & 47 & & $\mathrm{x}$ & & $\mathrm{hm}$ & an & & & \\
\hline & Portulaca $\mathrm{sp} .2$ & 66 & & $\mathrm{x}$ & & $\mathrm{hm}$ & an & & & \\
\hline
\end{tabular}




\begin{tabular}{|c|c|c|c|c|c|c|c|c|c|c|}
\hline \multirow[b]{2}{*}{ Famílias } & \multirow[b]{2}{*}{ Espécies } & \multicolumn{4}{|c|}{ Hábitat } & \multirow[t]{2}{*}{ FV } & \multirow[t]{2}{*}{ SD } & \multicolumn{3}{|c|}{$\begin{array}{l}\text { Locais de } \\
\text { ocorrência }\end{array}$} \\
\hline & & $\mathrm{NC}$ & $\mathbf{F}$ & D & $\mathbf{R}$ & & & 1 & 2 & 3 \\
\hline Rhamnaceae & Crumenaria decumbens Mart. & 44 & $\mathrm{x}$ & $\mathrm{x}$ & & th & an & $\mathrm{x}$ & & \\
\hline \multirow[t]{2}{*}{ Rubiaceae } & $\begin{array}{l}\text { Spermacoce capitata (Ruiz } \\
\text { \& Pav.) DC. }\end{array}$ & 126 & & $\mathrm{x}$ & & th & $\mathrm{au}$ & & & \\
\hline & Mitracarpus scabrellus Benth. & 127 & & $\mathrm{x}$ & & th & $\mathrm{au}$ & & & \\
\hline Sterculiaceae & Waltheria macropoda Turcz. & 77 & $\mathrm{x}$ & $\mathrm{x}$ & & $\mathrm{ch}$ & $\mathrm{au}$ & $\mathrm{x}$ & & \\
\hline Tiliaceae & Corchorus sp. & 149 & & $\mathrm{x}$ & & th & $\mathrm{au}$ & $\mathrm{x}$ & & \\
\hline Verbenaceae & Lantana camara $\mathrm{L}$. & 16 & $\mathrm{x}$ & & & fh & zo & $\mathrm{x}$ & & \\
\hline Vitaceae & Cissus simsiana Schult. \& Schult. f. & 26 & $\mathrm{x}$ & $\mathrm{x}$ & & $\mathrm{ch}$ & zo & & & \\
\hline Vochysiaceae & Callisthene fasciculata Mart. & 152 & $\mathrm{x}$ & $\mathrm{x}$ & & $\mathrm{ch}$ & $\mathrm{au}$ & & & \\
\hline
\end{tabular}

Tabela 2 - Comparação dos dados florísticos e climáticos de inselbergues em distintas localidades. Números de levantamentos (NL), espécies (NE), famílias (NF) e famílias mais representativas. Dados ambientais: altitude $(\mathrm{m})$, precipitação $(\mathrm{mm})$ e temperatura $\left({ }^{\circ} \mathrm{C}\right)$ média anual e número de meses secos $(\mathrm{ms}) . *$ dados não informados pelos autores.

\begin{tabular}{|c|c|c|c|c|c|c|c|c|}
\hline $\begin{array}{l}\text { Pais } \\
\text { (Região) }\end{array}$ & m & $\mathbf{m m}$ & ${ }^{\circ} \mathbf{C}$ & ms & NL & $\mathbf{N E}$ & $\mathbf{N F}$ & $\begin{array}{l}\text { Famílias mais representativas } \\
\text { (números de espécies ou porcentagens) }\end{array}$ \\
\hline $\operatorname{Brasil}(\mathrm{NE})^{1}$ & 270 & 868,5 & 26,6 & 7 & 1 & 77 & 41 & $\begin{array}{l}\text { Leguminosae (11), Poaceae (10), } \\
\text { Euphorbiaceae (5), Asteraceae e } \\
\text { Convolvulaceae (4) }\end{array}$ \\
\hline $\operatorname{Brasil}(\mathrm{NE})^{2}$ & 270 & $*$ & $*$ & $*$ & 1 & 48 & 32 & $\begin{array}{l}\text { Orchidaceae (5), Bromeliaceae (4) e } \\
\text { Euphorbiaceae (4) }\end{array}$ \\
\hline $\operatorname{Brasil}(\mathrm{NE})^{3}$ & $310-430$ & $*$ & $*$ & $*$ & 1 & 34 & 19 & Bromeliaceae (6) e Euphorbiaceae (4) \\
\hline $\operatorname{Brasil}(\mathrm{SE})^{4}$ & $20-1600$ & * & $15-24$ & 0 & 6 & 36 & 13 & $\begin{array}{l}\text { Bromeliaceae (10), Velloziaceae (5) e } \\
\text { Orchidaceae (4) }\end{array}$ \\
\hline $\operatorname{Brasil}(\mathrm{SE})^{5}$ & $10-1400$ & $*$ & $*$ & $*$ & 8 & 85 & 30 & $\begin{array}{l}\text { Bromeliaceae (16), Asteraceae (8) e } \\
\text { Velloziaceae (7) }\end{array}$ \\
\hline $\operatorname{Brasil}(\mathrm{SE})^{6}$ & 1600 & 1300 & 18 & $*$ & 1 & 55 & 23 & $\begin{array}{l}\text { Orchidaceae (14), Asteraceae (7) e } \\
\text { Cyperaceae (6) }\end{array}$ \\
\hline $\operatorname{Brasil}(\mathrm{SE})^{7}$ & 2400 & 2273 & 17,1 & $*$ & 2 & 114 & 48 & $\begin{array}{l}\text { Poaceae (17), Asteraceae (12), } \\
\text { Cyperaceae (6) e Melastomataceae (6) }\end{array}$ \\
\hline Bolívia $(E)^{8}$ & 1000 & $\begin{array}{l}1200- \\
2000\end{array}$ & $24-25$ & $4-2$ & 11 & 130 & 17 & $\begin{array}{l}\text { Poaceae, Bromeliaceae, Cactaceae, } \\
\text { Orchidaceae, Araceae e Cyperaceae }\end{array}$ \\
\hline Venezuela $(\mathrm{SE})^{9}$ & 500 & $\begin{array}{l}1015- \\
3054\end{array}$ & $\begin{array}{l}27,1- \\
27,5\end{array}$ & $5-0$ & 58 & 593 & 40 & $\begin{array}{l}\text { Cyperaceae e Rubiaceae (40), } \\
\text { Melastomataceae (36), Orchidaceae (33) } \\
\text { e Poaceae (31) }\end{array}$ \\
\hline Guiana Francesa ${ }^{10}$ & $200-410$ & $\begin{array}{l}2250- \\
3250\end{array}$ & $*$ & 5 & 3 & 78 & 37 & $\begin{array}{l}\text { Cyperaceae }(10 \%) \text {, Poaceae }(9 \%) \text { e } \\
\text { Lentibulariaceae }(6 \%)\end{array}$ \\
\hline $\begin{array}{l}\text { Narrogin, } \\
\text { Austrália }(\mathrm{W})^{11}\end{array}$ & $*$ & $*$ & $*$ & $*$ & 6 & 238 & 54 & $\begin{array}{l}\text { Proteaceae (21), Orchidaceae (19), } \\
\text { Asteraceae e Myrtaceae (17), Poaceae } \\
\text { (16) e Fabaceae (13) }\end{array}$ \\
\hline $\begin{array}{l}\text { Costa do } \\
\operatorname{Marfim}(\mathrm{N})^{12}\end{array}$ & $200-470$ & $\begin{array}{l}900- \\
1600\end{array}$ & $\begin{array}{l}26- \\
27,3\end{array}$ & 6 & 18 & 34 & 15 & $\begin{array}{l}\text { Cyperaceae (9), Poaceae (5) e } \\
\text { Scrophulariaceae (3) }\end{array}$ \\
\hline
\end{tabular}




\begin{tabular}{|c|c|c|c|c|c|c|c|c|}
\hline $\begin{array}{l}\text { Pais } \\
\text { (Região) }\end{array}$ & m & $\mathbf{m m}$ & ${ }^{\circ} \mathbf{C}$ & ms & NL & $\mathbf{N E}$ & $\mathbf{N F}$ & $\begin{array}{l}\text { Famílias mais representativas } \\
\text { (números de espécies ou porcentagens) }\end{array}$ \\
\hline $\operatorname{Namíbia}(\mathrm{N})^{13}$ & $\begin{array}{l}840- \\
1014\end{array}$ & $20-50$ & $\begin{array}{l}17,6- \\
24\end{array}$ & $*$ & 2 & 82 & 30 & $\begin{array}{l}\text { Asteraceae (13), Poaceae (8) e } \\
\text { Euphorbiaceae (5) }\end{array}$ \\
\hline $\begin{array}{l}\text { Costa do } \\
\operatorname{Marfim}(\mathrm{NE})^{14}\end{array}$ & $200-300$ & $\begin{array}{l}900- \\
1200\end{array}$ & $26-28$ & 6 & 1 & 26 & 17 & $\begin{array}{l}\text { Poaceae (6), Cyperaceae (3), } \\
\text { Scrophulariaceae (2) e Lentibulariaceae (2) }\end{array}$ \\
\hline Costa do Marfim ${ }^{15}$ & $*$ & 1833 & $*$ & $2-4$ & 8 & 66 & 35 & $\begin{array}{l}\text { Poaceae e Cyperaceae (5), Acanthaceae } \\
\text { (4), Commelinaceae (4) e Malvaceae (4) }\end{array}$ \\
\hline Costa do Marfim ${ }^{16}$ & $*$ & $\begin{array}{l}900- \\
2300\end{array}$ & $6-0$ & $*$ & 11 & 15 & 10 & $\begin{array}{l}\text { Cyperaceae (3), Orchidaceae (2), } \\
\text { Poaceae (2) e Rubiaceae (2) }\end{array}$ \\
\hline
\end{tabular}

1. Quixadá; 2. França et al. (2005); 3. França et al. (2006); 4. Porembeski et al. (1998); 5. Meirelles et al. (1999); 6. Caiafa \& Silva (2005); 7. Ribeiro et al. (2007); 8. Ibisch et al. (1995); 9. Gröger \& Barthlott (1996); 10. Sarthou \& Villiers (1998); 11. Pigott \& Sage (1997); 12. Krieger et al. (2000); 13. Burke et al. (1998); 14. Porembski \& Barthlott (1997); 15. Porembski et al. (1996); 16. Porembski et al. (1995).

Tabela 3 - Porcentagem das síndromes de dispersão das espécies de inselbergues em Quixadá (1), Sudeste do Brasil (2. Porembski et al. 1998), e Deserto da Namíbia ( 3. Burke et al. 1998 e 4. Burke et al. 1998). Síndromes de dispersão: zoocoria (zo), anemocoria (an), autocoria (au), hidrocoria (hi). Espécies sem determinação da síndrome (sd). * = ausência de informações.

\begin{tabular}{llllll}
\hline País (Região) & \multicolumn{5}{c}{ Síndromes de dispersão (\%) } \\
& zo & an & au & hi & sd \\
\hline Brasil (NE) & 16 & $\mathbf{4 9}$ & 35 & $*$ & $*$ \\
Brasil (SE) $^{1}$ & 16,6 & $\mathbf{5 8 , 3}$ & 5,5 & 0 & 0 \\
Deserto da Namíbia $^{3}$ & 10 & $\mathbf{5 2 , 6}$ & 13,5 & 4,5 & 15,7 \\
Deserto da Namíbia $^{4}$ & 12,7 & $\mathbf{3 9 , 8}$ & 16,9 & 11 & 19,5 \\
\hline
\end{tabular}

pelas famílias Leguminosae e Euphorbiaceae (Tab. 1). Entre as espécies zoocóricas, destacamse as das famílias Cactaceae e Myrtaceae, com três e duas espécies cada (Tab. 1). A família Mytaceae não foi registrada na caatinga do entorno, porém, há áreas serranas relativamente próximas, como a serra de Baturité, com flora rica em Myrtaceae (Araújo et al. 2007), que podem funcionar como uma das fontes de propágulos.

A dominância de síndromes abióticas entre as espécies do monólito de Quixadá (Tab. 3) confirma o padrão esperado para caatinga (ver Griz \& Machado 2001; Barbosa et al. 2002). Além disso, conforme verificado nos trabalhos de Burke et al. (1998) e Porembski et al. (1998), esses tipos de síndrome possivelmente constituem um dos atributos dominantes entre as espécies que colonizam ilhas de afloramentos rochosos, independente do clima. As síndromes abióticas, principalmente a anemocoria, representam mais de $80 \%$ da flora dos inselbergues (ver Porembski et al. 1998; Burke et al. 1998) (Tab.3). Até naqueles situados sob climas úmidos, como nos encravado na floresta atlântica do Sudeste do Brasil, há predomínio de síndromes abióticas (Porembski et al. 1998). Assim, habitats sob maior exposição aos ventos e à insolação (Barthlott et al. 1993; Ibisch et al. 1995; Porembski et al. 1998), mesmo sob domínio climático úmido, devem favorecer a entrada e a colonização de espécies de síndromes abióticas.

As formas de vida encontradas na área deste estudo foram: $34(44,2 \%)$ terófitos, $19(24,7 \%)$ fanerófitos, $11(14,6 \%)$ caméfitos, $10(13,4 \%)$ hemicriptófitos, 2 (2,6\%) criptófitos geófitos e $1(1,2 \%)$ aerófito. Este espectro foi similar ao da flora do entorno (Tab. 4). A alta porcentagem de espécies anuais (terófitos) em Quixadá 
Tabela 4 - Espectro biológico das espécies do inselbergue de Quixadá, caatinga do entorno e dos inselbergues de outras regiões geográficas. Formas de vida: $\mathrm{fn}=$ fanerófito, $\mathrm{ch}=$ caméfito, $\mathrm{hm}=$ hemicriptófito, $\mathrm{cg}=$ criptófito geófito, th=terófito, ar=aerófito. *= ausência de informações.

\begin{tabular}{|c|c|c|c|c|c|c|c|}
\hline \multirow{2}{*}{$\begin{array}{l}\text { Estado ou } \\
\text { Região, País }\end{array}$} & \multirow[b]{2}{*}{ fn } & \multirow[b]{2}{*}{ ch } & \multicolumn{3}{|c|}{ Formas de Vida (\%) } & \multirow[b]{2}{*}{ ar } & \multirow[b]{2}{*}{ Outras formas } \\
\hline & & & hm & cg & th & & \\
\hline $\mathrm{CE}$, Brasil $^{1}$ & 24,7 & 14,6 & 13,4 & 2,6 & 44,2 & 1,2 & $*$ \\
\hline Entorno-CE, Brasil ${ }^{2}$ & 23,6 & 15,8 & 12,8 & 2,3 & 42,9 & $*$ & $*$ \\
\hline $\mathrm{BA}$, Brasil $^{3}$ & 54 & 12,5 & 8,3 & 6,3 & 6,3 & $*$ & Liana: 10 \\
\hline $\mathrm{BA}, \mathrm{Brasil}^{4}$ & 64 & 6 & 9 & 0,0 & 12 & $*$ & Epífita: 9 \\
\hline RJ, Brasil ${ }^{5}$ & 45 & 0,0 & 45 & 5 & 0,0 & $*$ & Epífita: 5 \\
\hline RJ, Brasil ${ }^{5}$ & 31 & 0,0 & 48 & 3,4 & 0,0 & $*$ & Epífita: 13,8, Hemiepífita: 3,4 \\
\hline RJ, Brasil ${ }^{5}$ & $\mathbf{5 0}$ & 0,0 & 50 & 0,0 & 0,0 & $*$ & 0,0 \\
\hline RJ, Brasil ${ }^{5}$ & 40,7 & 3,7 & 44,4 & 3,7 & 0,0 & $*$ & Epífita: 3,7, Hemiepífita: 3,7 \\
\hline RJ, Brasil ${ }^{5}$ & 11 & 0,0 & $\mathbf{7 7 , 7}$ & 11 & 0,0 & $*$ & 0,0 \\
\hline RJ, Brasil ${ }^{5}$ & 38,8 & 0,0 & 50 & 11,2 & 0,0 & $*$ & 0,0 \\
\hline RJ, Brasil ${ }^{5}$ & $\mathbf{5 0}$ & 0,0 & 38,5 & 0,0 & 11 & $*$ & 0,0 \\
\hline RJ, Brasil ${ }^{5}$ & 33,3 & 7,4 & 48 & 3,7 & 0,0 & $*$ & Epífita: 3,7, Hemiepífita: 3,7 \\
\hline RJ, Brasil ${ }^{6}$ & 31,8 & 2,3 & 43,5 & 4,7 & 2,3 & $*$ & $*$ \\
\hline RJ, Brasil ${ }^{7}$ & 29,8 & 15,8 & 36,8 & 15,8 & 1,8 & $*$ & $*$ \\
\hline MG, Brasil $^{8}$ & 12,7 & 29 & 41,8 & 5,5 & 10,9 & $*$ & $*$ \\
\hline NE, Guiana Francesa ${ }^{9}$ & 16 & 21 & 41 & 0,0 & 12 & $*$ & Liana: 10 \\
\hline Costa do Marfim ${ }^{10}$ & 8,8 & 2,9 & 5,8 & 0,0 & 73,5 & $*$ & Hidrófita: 2,9, Epífita: 2,9 \\
\hline Costa do Marfim ${ }^{11}$ & 0,0 & 7,7 & 7,7 & 7,7 & 77 & $*$ & $*$ \\
\hline Costa do Marfim ${ }^{12}$ & 16,6 & 6 & 3 & 6 & 40,9 & $*$ & Epífita/litófita: 6Perenes: 16,6 \\
\hline Costa do Marfim ${ }^{13}$ & 6,6 & 20 & 6,6 & 6,6 & 60 & $*$ & $*$ \\
\hline
\end{tabular}

1. Quixadá; 2. Costa et al. (2007); 3. França et al. (2005); 4. França et al. (2006); 5. Meirelles et al. (1999); 6. Safford \& Martinelli (2000); 7. Ribeiro et al. (2007); 8. Caiafa \& Silva (2005); 9. Sarthou \& Villiers (1998); 10. Krieger et al. (2000); 11. Porembski et al. (1995); 12. Porembski et al. (1996); 13. Porembski \& Barthlott (1997).

confirma o padrão esperado para a flora da caatinga (Araújo et al. 2005b; Costa et al. 2007; Rodal et al. 2005).

Os terófitos são a forma de vida dominante nos afloramentos rochosos de regiões áridas e semi-áridas (Tab. 4), as quais também apresentam flora com espécies predominantemente dispersas por fatores abióticos, principalmente o vento, conforme já relatado nas diversas publicações de Porembski e colaboradores. O fato das áreas estudadas por França et al. (2005 e 2006), no domínio semi-árido da Bahia, não se encaixam neste padrão (Tab. 4), possivelmente, se deve a peculiaridades de variáveis abióticas locais, como o solo, e a vegetação circundante. Assim, este estudo confirma o padrão esperado para os atributos síndromes de dispersão e forma de vida de vegetação sob climas áridos e semi-áridos.

\section{Agradecimentos}

Aos revisores anônimos que contribuíram para a melhor qualidade deste manuscrito.

\section{REFERÊNCIAS BibLIOGRÁFICAS}

Araújo, F. S.; Rodal, M. J. N.; Barbosa, M. R. V. \& Martins, F. R. 2005a. Repartição da flora lenhosa no domínio da Caatinga. In: Araújo, F. S.; Rodal, M. J. N. \& Barbosa, M. R. V. (orgs). Análise das variações da biodiversidade do bioma Caatinga: suporte a estratégias regionais de conservação. Ministério do Meio Ambiente. Brasília. p. 17-35.

Araújo, F. S.; Costa, R. C.; Figueiredo, M. A. \& Nunes, E. P. 2005b. Vegetação e flora fanerogâmica da área Reserva Serra das Almas, Ceará. In: Araújo, F. S.; Rodal, M. J. N. \& Barbosa, M. R. V. (orgs). 
Análise das variações da biodiversidade do bioma Caatinga: suporte a estratégias regionais de conservação. Ministério do Meio Ambiente. Brasília. p. 91-119.

Araújo, F. S.; Gomes, V. S.; Lima-Verde, L. W.; Figueiredo, M. A.; Bruno, M. M. A.; Nunes, E. P.; Otutumi, A. T. \& Ribeiro, K. A. 2007. Efeito da variação topoclimática na composição e riqueza da flora fanerogâmica da serra de Baturité, Ceará. In: Oliveira, T. S. \& Araújo, F. S. (orgs). Diversidade e conservação da biota da serra de Baturité, Ceará. Seri\&A Gráfica, Fortaleza. Pp. 137-162.

Barbosa, D. C. A.; Silva, P. G. G. \& Barbosa, M. C. A. 2002. Tipos de frutos e síndromes de dispersão de espécies lenhosas da Caatinga de Pernambuco. In: Tabarelli, M. \& Silva, J. M. C. (orgs). Diagnóstico da biodiversidade de Pernambuco. Pp. 609-617.

Barthlott, W.; Gröger, A \& Porembski, S. 1993. Some remarks on the vegetation of tropical inselbergs: diversity and ecological differentiation. Biogeographica 69(3): 105-124.

Brummitt, R.K. \& Powell, C.E. 1992. Authors of plant names. Royal Botanic Gardens, Kew, 732p.

Burke, A.; Jürgens, N. \& Seely, M. K. 1998. Floristic affinities of an inselberg archipelago in the southern Namib desert - relic of the past, centre of endemism or nothing special? Journal of Biogeography 25: 311-317.

Caiafa, A. N. \& Silva, A. F. 2005. Composição florística e espectro biológico de um campo de altitude no Parque Estadual da Serra do Brigadeiro, Minas Gerais - Brasil. Rodriguésia 56(87): 163-173.

Costa, R. C.; Araújo, F. S. \& Limaverde, L. W. 2007. Flora and life-form spectrum in an area of deciduous thorn woodland (caatinga) in northeastern, Brazil. Journal of Arid Environments 68(2): 237-247.

Cronquist, A. 1981. An integrated system of classification of flowering plants. Columbia University Press, New York, $1262 \mathrm{p}$.
França, F.; Melo, E. \& Santos, C. C. 1997. Flora de inselbergs da região de Milagres, Bahia, Brasil: caracterização da vegetação e lista de espécies de dois inselbergs. Sitientibus Série Ciências Biológicas 17: 163-184

França, F.; Melo, E. \& Gonçalves, J. M. 2006. Aspectos da diversidade da vegetação de um inselbergue no semi-árido da Bahia, Brasil. Stientibus Série Ciências Biológicas 6 (1): 30-35

França, F.; Melo, E.; Santos, A. K. A.; Melo, J. G. A. N.; Marques, M.; Silva-Filho, M. F. B.; Moraes, L. \& Machado, C. 2005. Estudo ecológico e florístico em ilhas de vegetação de um inselberg no semi-árido da Bahia, Brasil. Hoehnea 32 (1): 93-101.

Fundação Cearense de Meteorologia e Recursos Hídricos (FUNCEME). 2007. Disponível em: <http://www.funceme. com.br> Acesso em:13 jun. 2007.

Galán de Mera, A.; Hagen, M. A. \& Vicente Orellana, J. A. 1999. Aerophyte, a new life form in Raunkiaer's classification. Journal of Vegetation Science 10: 65-68.

Griz, L. M. S. \& Machado, I. C. S. 2001. Fruiting phenology and seed dispersal syndromes in caatinga, a tropical dry forest in northeast of Brazil. Journal of Tropical Ecology 17: 303-321

Gröger, A. \& Barthlott, W. 1996. Biogeography and diversity of the inselberg (Laja) vegetation of southern Venezuela. Biodiversity Letters 3: 165-79.

Guerra, A. T. 1978. Dicionario geológicogeomorfológico. Fundação Instituto Brasileiro de Geografia e Estatística (IBGE), Rio de Janeiro. 446p.

Ibisch, P. L.; Rauer, G.; Rudolph, D. \& Barthlott W. 1995. Floristic, biogeographical, and vegetational aspects of Pre-Cambrian rock outcrops (inselbergs) in eastern Bolivia. Flora 190: 299-314.

Kovács-Lang, E.; Kroel-Dulay, G.; Kertész, M.; Fekete, G.; Bartha, S.; Mika, J.; DobiWantuch, I.; Rédei, T.; Rajkai K. \& Hahn, I. 2000. Changes in composition of sand grasslands along a gradient in Hungary and 
implications for climate change. Phytocoenologia 30 (3-4): 385-407.

Krebs, C. J. 1989. Ecological methodology. Harper Collins, New York. 620p.

Krieger, A.; Porembisk, S. \& Barthlott, W. 2000. Vegetation of seasonal rock pools on inselbergs situated in the savanna zone of the Ivory Coast (West Africa). Flora 195: 257-266.

Lewis, G. D.; Schrire, B. D.; Mackinder, B. A. \& Lock, J. M. 2005. Legumes of the world. Royal Botanic Garden, Kew, 577p.

Mamede, M. A. \& Araújo, F. S. 2008. Effects of slash and burn practices on a soil seed bank of caatinga vegetation in Northeastern Brazil. Journal of Arid Environments 72: 458-470.

Meirelles, S. T.; Pivello, V. R. \& Joly, C. A. 1999. The vegetation of granitic orck outcrop in Rio de Janeiro, Brazil, and the need for its protection. Environmental Conservation 26 (1): 10-20.

Mori, S.; Matos-Silva, L.; Lisboa, G. \& Coradin, L. 1989. Manual de manejo do herbário fanerogâmico. $2^{\mathrm{a}}$ ed. Ilhéus. Centro de Pesquisas do Cacau. 104p.

Pigot, J. P. \& Sage, L. W. 1997. Remmnant vegetation, priority flora and weed invasions at Yilliminning Rock, Narrogin, Western Australia. Journal of the Royal Society of Western Australia 80: 201-208.

Porembski, S. \& Barthlott, W. 1997. Seazonal dynamics of plant diversity on inselbergs in the Ivory Coast (West Africa). Botanical Acta 110: 466-472.

Porembski, S. \& Barthlott, W. 2000. Granitic and gneissic outcrops (inselbergs) as centers of desiccation-tolerant vascular plants. Plant Ecology 151: 19 - 28.

Porembski, S.; Barthlott, W.; Dörrstock, S. Biedinger, N. 1994 Vegetation of rock outcrops in Guinea: granite inserlbergs, sandstone table mountains and ferricretes - remarks on species numbers and endemism. Flora 189 (4): 315-326.

Porembski, S.; Brown, G. \& Barthlott, W. 1995. A species-poor tropical sedge community: Afrotrilepis pilosa mats on inselbergs in West Africa. Nordic Journal of Botany 16 (3): 239-245.

Porembski, S.; Fischer, E. \& Biedinger, N. 1997. Vegetation of inselbergs, quarzitic outcrops and ferricretes in Rwanda and eastern Zaire (Kivu). Bulletin du Jardin Botanique National de Belgique 66: 81-99.

Porembski, S.; Martinelli, G.; Ohlemüller, R. \& Barthlott, W. 1998. Diversity and ecology of saxicolous vegetation mats on inselbergs in the Brazilian Atlantic rainforest. Diversity and Distributions 4: 107-119.

Porembski, S.; Szarzynsk, J.; Mund, Jan-Petter \& Barthlott, W. 1996. Biodiversity and vegetation of small-sized inselbegs in a West africa rain forest (Täi, Ivory Coast). Journal of Biogeography 23: 47-55.

Raunkiaer, C. 1934. The life forms of plants and statistical plant geography. Clarendon Press, Oxford, 632p.

Ribeiro, K. T.; Medina, B. M. O. \& Scarano, F. R. 2007. Species composition and biogeographic relations of the rock outcrop flora on the higth plateau of Itatiaia, SEBrazil. Revista Brasileira de Botânica 30(4): 623-639.

Rodal, M. J. N.; Lins e Silva, A. C. B.; Pessoa, L. M. \& Cavalcanti, A. D. C. 2005. Vegetação e flora fanerogâmica da área de Betânia, Pernambuco. In: Araújo, F. S.; Rodal, M. J. N. \& Barbosa, M. R. V. (orgs). Análise das variações da biodiversidade do bioma Caatinga: suporte a estratégias regionais de conservação. Ministério do Meio Ambiente, Brasília. Pp. 140-166.

Sarthou, C. \& Villiers, Jean-François. 1998. Epilithic plant communities on inselbergs in French Guiana. Journal of Vegetation Science 9: 847-860.

Scarano, F. R. 2007. Rock outcrop vegetation in Brazil. a brief overview. Revista Brasileira de Botânica 30(4): 561-568.

Souza, M. J. N. 1988. Contribuição ao estudo das unidades morfo-estruturais do estado do Ceará. Revista de Geologia 1: 73-91.

SUDENE. 1982. Climatologia e balanço hídrico do Ceará: dados pluviométricos, temperaturas 
estimadas por regressão. Relatório Técnico Projeto: Delimitação e Regionalização do Brasil semi-árido, 32p.

van der Pijl, L. 1982. Principles of dispersal in higher plants. $2^{\text {nd }}$ ed. Springer Verlag, Berlin, 215p. van Rooyen, M. W.; Theron, G.K. \& Grobbelaar, N. 1990. Life forms and spectra of flora of Namaqualand, South Africa. Journal of Arid Environments 19: 133-145. 